\title{
Pregnancy after myocardial infarction and coronary artery bypass grafting - is it safe?
}

\author{
Agnieszka Janion-Sadowska ${ }^{1}$, Marcin Sadowski ${ }^{1}$, Łukasz Zandecki ${ }^{1}$, Jacek Kurzawski ${ }^{1}$, Anna Polewczyk ${ }^{1}$, \\ Marianna Janion ${ }^{1,2}$ \\ 'Świętokrzyskie Cardiology Center, Provincial Hospital Complex, Kielce, Poland \\ ${ }^{2}$ Faculty of Health Sciences, The Jan Kochanowski University, Kielce, Poland
}

Postep Kardiol Inter 2014; 10, 1 (35): 29-31 DOI: 10.5114/pwki.2014.41464

\begin{abstract}
A bstract
Pregnancy after myocardial infarction ( $\mathrm{MI})$ and coronary artery bypass grafting (CABG), although still rare, is slowly becoming a challenge in everyday clinical practice. In recent decades $\mathrm{MI}$ has been observed to occur more frequently in young women. Concurrently an increasing number of women decide to become pregnant at more advanced age. Although pregnancy after MI and CABG may be possible and safe, a multidisciplinary approach involving careful evaluation by the cardiac and obstetric team in each individual is mandatory. Two cases of pregnant women with a history of MI treated with CABG are presented and their management is discussed.
\end{abstract}

Key words: pregnancy, myocardial infarction, coronary artery bypass grafting.

\section{Introduction}

Young women are generally at low risk of serious cardiac conditions and contribute relatively little to the overall number of coronary artery disease (CAD) patients. Cardiovascular disease occurs in $0.4-4.1 \%$ of pregnant women whereas CAD complicates only $0.01 \%$ of all pregnancies [1]. The risk profile of women nowadays differs significantly from that of 50 years ago. Women frequently smoke, do managerial jobs, postpone pregnancy and use oral contraceptives. When they finally decide to become pregnant they are at high risk of CAD or may even have had the first episode of myocardial infarction (MI). Hypercoagulability, changes in blood volume, heart rate and cardiac output increase myocardial oxygen demand, which may induce angina or heart failure. Birth-related pain, effort and blood pressure alterations also predispose to complications related to myocardial ischemia [1-3]. Although MI during pregnancy has been reported in many cases and the management in this situation is clearly presented in current guidelines [3], data on pregnancy after $\mathrm{Ml}$ are scarce, especially in women with multivessel disease that required comprehensive surgical revascularization. A multidisciplinary approach involving careful evaluation by the cardiac and obstetric team in each individual is mandatory in such cases.

We report 2 cases of pregnant women with a history of $\mathrm{Ml}$ treated with coronary artery bypass grafting (CABG).

\section{Case reports}

\section{Case 1}

This 38-year-old woman after 2 caesarean sections at the age of 36 and 37 developed $\mathrm{MI}$ on the $15^{\text {th }}$ day postpartum after the second pregnancy, complicated by ventricular arrhythmia. Coronary angiography revealed double vessel disease - critical 95\% stenosis in the middle portion of the left anterior descending artery, and long $70-90 \%$ stenosis of the proximal circumflex artery. Coronary artery bypass grafting was performed on the $16^{\text {th }}$ day postpartum [4]. Ten months after CABG at 10 weeks pregnant with her third baby the medications were arbitrarily discontinued by the patient (acetylsalicylic acid - ASA, atorvastatin, angiotensin-converting enzyme inhibitor, and sotalol at 12 weeks). The pregnancy, delivery and postpartum course were uneventful, and a healthy, full-term infant weighing $3450 \mathrm{~g}$ was born

\section{Corresponding author:}

Łukasz Zandecki MD, Świętokrzyskie Cardiology Center, Provincial Hospital Complex, 2/3 Połowniaka St, 25-634 Kielce, Poland, phone: +48 606620 729, e-mail: lukasz.zandecki@gmail.com

Received: 2.12.2013, accepted: 9.01.2014. 
without congenital defects. Bottle feeding was recommended and pharmacotherapy was resumed with ASA $75 \mathrm{mg}$, atorvastatin $40 \mathrm{mg}$ and metoprolol succinate $50 \mathrm{mg}$. The patient has remained stable over 2 years of follow-up, and the baby is developing normally. Additional tests revealed normal lipid profile. An echocardiogram revealed mild left ventricular dysfunction (ejection fraction of $45 \%)$. The Bruce treadmill test demonstrated good exercise tolerance, without angina or ischemic ECG changes.

\section{Case 2}

This 38-year-old woman with 5 children, and familial hypercholesterolemia diagnosed at the age of 25 , was on chronic treatment with statins. At the age of 28 at 8 weeks pregnant with her fourth baby she discontinued atorvastatin $40 \mathrm{mg}$. The pregnancy was complicated by pregnancy-induced hypertension, and placental abruption at delivery. The patient developed postpartum genital tract infection and bleeding complications. Four years later at age 32 she suffered MI. Coronary angiography revealed multi-vessel disease, and the patient underwent surgical revascularization (CABG). In 2006, 5 years after the $\mathrm{MI}$ and $C A B G$, she became pregnant with her fifth baby. Until the $6^{\text {th }}$ week of pregnancy she had been receiving atorvastatin $80 \mathrm{mg}$ and acetylsalicylic acid $75 \mathrm{mg}$. Additional tests revealed total cholesterol (TC) $490 \mathrm{mg} / \mathrm{dl}$, HDL 45 mg/dl, LDL 397 mg/dl, triglycerides (TG) 241 mg/ dl. Serum lipids prior to delivery were as follows: TC 828 mg/dl, HDL 85 mg/dl, LDL 493 mg/dl, TG 623 mg/dl. The pregnancy, delivery (caesarean section) and postpartum course were uneventful. A healthy baby without congenital abnormalities was born at 38 weeks' gestation with $3050 \mathrm{~g}$ birth weight. Because of high cholesterol values bottle feeding was recommended, and pharmacotherapy with rosuvastatin $40 \mathrm{mg}$, ezetimibe $10 \mathrm{mg}$, ASA $75 \mathrm{mg}$, and bisoprolol $2.5 \mathrm{mg}$ was immediately instituted. The patient and her baby remain in a good state over follow-up; her lipid profile at 3 months of treatment: TC 387 mg/dl, LDL 306 mg/dl, HDL 24 mg/dl, TG 286 mg/dl.

\section{Discussion}

Pre-pregnancy risk assessment plays a crucial role in the management of women with a history of MI and CABG. Resting and exercise ECG and echocardiogram as well as coronary angiography should not be avoided. Women with significantly decreased ejection fraction, heart failure symptoms, angina pectoris or untreated advanced changes in coronary arteries should be discouraged from getting pregnant. Life-style changes including smoking cessation, dietary habits and physical activity are also important in decreasing risk in this subset of patients.

Pharmacotherapy typical for post-MI patients, although beneficial in general, is not widely recommended in pregnant women due to its harmful potential or estab- lished teratogenicity. Low doses of ASA seem to be safe in pregnancy (category $\mathrm{C}$ ); teratogenicity has not been noted so far when used in cardiac doses $[3,5]$. Our 2 patients after CABG had been receiving ASA $75 \mathrm{mg}$ in the first 6-8 weeks in pregnancy and then they arbitrarily decided to discontinue treatment. Both women gave birth to healthy babies. It should however be emphasized that safety of ASA in early pregnancy has not been unequivocally established. In our pregnant patients after CABG, with stable angina pectoris and preserved left ventricular contractility prior to pregnancy, no negative effects of using ASA in early pregnancy and no short-term consequences of subsequent ASA discontinuation until delivery were observed.

$\beta$-Adrenergic blockers reduce mortality after $\mathrm{MI}$ and are recommended as relatively safe drugs for pregnant women [3]. Our patient (case 2) discontinued sotalol at 12 weeks pregnant without a negative effect on the further course of pregnancy. In patients after effective myocardial revascularization and without significant postinfarction myocardial injury, discontinuation of optimal pharmacotherapy during pregnancy is unlikely to result in decompensation of the cardiovascular system, worsening angina symptoms or ventricular arrhythmias. Most $\beta$-blockers pass through the placenta and into breast milk and, although without proven teratogenicity, may induce bradycardia and hypoglycemia in fetuses [2].

Statins have proven efficacy in reducing cardiovascular risk with an unclear effect on the human fetus. According to current guidelines, they should be withdrawn in pregnancy, even as early as 3 months before planning to become pregnant and even in women with primary hypercholesterolemia, which should not have a negative impact on the CAD course $[3,6,7]$. There are some controversial opinions on the negative effects of statins in pregnancy and perhaps the recommendation will be revisited when the results of the ongoing studies are available $[2,7,8]$. Both our patients had been using statins until 6-8 weeks pregnant, that is during the period of intense organogenesis, but so far no adverse effects either on the children or on the mothers have been found.

Angiotensin-converting enzyme inhibitors (ACE-I) are beneficial especially in a high-risk population after $\mathrm{MI}$ with arterial hypertension, heart failure and diabetes. However, pregnancy category D limits their use. Some studies suggest that ACE-I used in the first trimester do not significantly increase the risk of congenital defects in children, although it cannot be excluded that they increase the rate of miscarriage [2]. One woman in our study (case 1) had been using perindopril $2.5 \mathrm{mg}$ until 8 weeks pregnant but no adverse effects were noted.

\section{Conclusions}

Pregnancy in patients after MI treated with CABG and with normal left ventricular ejection fraction seems 
to be possible and safe, which was corroborated in our study. Early comprehensive surgical revascularization in multivessel disease in our 2 patients provided the setting for normal subsequent pregnancy at 10 months and at 5 years after CABG. In such patients discontinuation of optimal coronary pharmacotherapy during pregnancy is unlikely to have a negative impact on maternal health and is a safer approach for the fetus. Nevertheless, small doses of ASA and most $\beta$-blockers are considered to be relatively safe in pregnancy and may be used if necessary. In contrast, women with significantly decreased left ventricular ejection fraction, symptomatic heart failure or persisting abnormalities in coronary arteries should be discouraged from pregnancy.

\section{References}

1. Frenkel Y, Barkai G, Reisin L, et al. Pregnancy after myocardial infarction: are we playing safe? Obstet Gynecol 1991; 77: 822-825.

2. Janion-Sadowska A, Sadowski M, Kurzawski J, et al. Pregnancy after acute coronary syndrome: a proposal for patients' management and a literature review. Biomed Res Int 2013; 2013: 957027.

3. European Society of Gynecology; Association for European Paediatric Cardiology; German Society for Gender Medicine; Authors/ Task Force Members, Regitz-Zagrosek V, BlomstromLundqvist C, Borghi C, et al. ESC Guidelines on the management of cardiovascular diseases during pregnancy: the Task Force on the Management of Cardiovascular Diseases during Pregnancy of the European Society of Cardiology (ESC). Eur Heart J 2011; 32: 3147-3197.

4. Polewczyk A, Sielski J, Janion-Sadowska A, et al. Acute myocardial infarction - multivessel coronary artery disease in a 37-year-old postpartum women. Kardiol Pol 2009; 67: 1381-1384.

5. De Santis M, De Luca C, Mappa I, et al. Clopidogrel treatment during pregnancy: a case report and a review of literature. Intern Med 2011; 50: 1769-1773.

6. Ito MK, McGowan MP, Moriarty PM; National Lipid Association Expert Panel on Familial Hypercholesterolemia. Management of familial hypercholesterolemias in adult patients: recommendations from the National Lipid Association Expert Panel on Familial Hypercholesterolemia. J Clin Lipidol 2011; 5: S38-S45.

7. Ofori B, Rey E, Bérard A. Risk of congenital anomalies in pregnant users of statin drugs. Br J Clin Pharmacol 2007; 64: 496-499.

8. Lecarpentier E, Morel O, Fournier T, et al. Statins and pregnancy: between supposed risks and theoretical benefits. Drugs 2012; 72: 773-788. 